\title{
Development of Reconfigurable Five-axis Machine Tool Using OPEN Computer Numerical Control System Integration Architecture
}

\author{
Yuan-Ming Cheng ${ }^{1 *}$ and Mu-Sheng Lin $^{2}$ \\ ${ }^{1}$ Department of Intelligent Robotics, National Pingtung University, \\ Pingtung 90004, Taiwan, Republic of China \\ ${ }^{2}$ Department of Mechanical \& Automation Engineering, Kao Yuan University, \\ Kaohsiung City 821, Taiwan, Republic of China
}

(Received July 7, 2020; accepted November 2, 2020)

Keywords: parallel mechanism, five-axis machine tool, OPEN CNC, postprocessing

The purpose of this study is to use an OPEN computer numerical control (CNC) system to develop a reconfigurable five-axis machine tool (RFMT) for system integration research. The RFMT is mainly composed of a three-degree-of-freedom (3DOF) parallel platform and an $X-Y$ table. In the study, the human-machine interface of OPEN CNC was written, and the programmable logic controller (PLC) architecture of OPEN CNC is used to control threeaxis simultaneous movement or individual drives. The processing path of this study uses a postprocessing program to transfer the numerical control (NC) code with five-axis machining software, and the $Z, \alpha$, and $\beta$ values of the $\mathrm{NC}$ program are used to convert each axis value of a three-axis motion platform by inverse kinematics. Finally, rough and finishing methods are used to cut a concave circle to verify the accuracy of RFMT.

\section{Introduction}

With the increase in the quality of life and advancement of technologies, the requirements for product performance and quality have also increased. Industries tend to develop products that are diversified, refined, personalized, comfortable, and environmentally friendly. Conventional three-axis machines have become insufficient for application to the increasingly complex surface machining required in the growing computer, communication, and consumer electronics industries as well as in aerospace, biomedicine, and mold development. Five-axis machining developed in recent years has gradually matured. It is characterized by high rigidity, precision, speed, and production efficiency as well as the capability to machine multisurface composite workpieces in a single setup. Therefore, five-axis machining has been gradually applied to mass production, and the demand for five-axis machine centers has substantially increased.

A reconfigurable parallel five-axis machine center comprises a three-degree-of-freedom (3DOF) parallel platform and an $X-Y$ table (Fig. 1). The 3DOF parallel platform can be

*Corresponding author: e-mail: chengym@mail.nptu.edu.tw https://doi.org/10.18494/SAM.2020.3099 


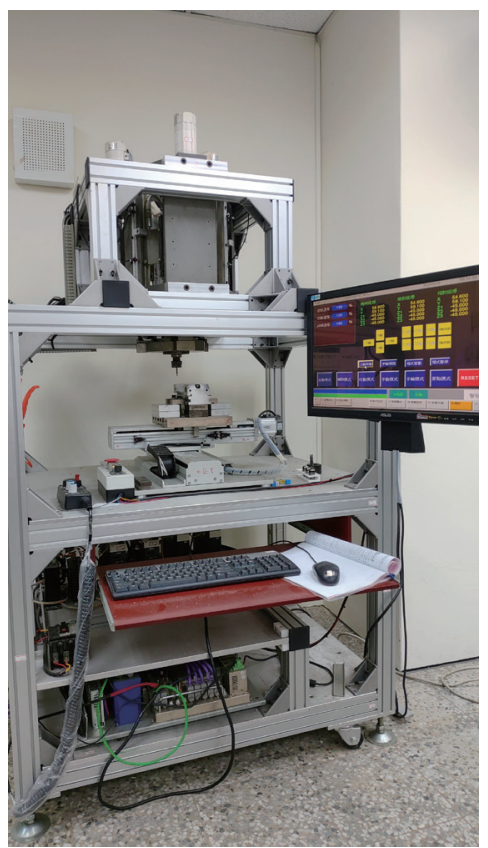

Fig. 1. (Color online) Parallel five-axis machining center.

divided into three types according to how the actuator axes are installed. In the first type, the actuator axes are installed vertically; ${ }^{(1)}$ in the second type, the three actuator axes are parallel to each other; ${ }^{(2)}$ in the third type, the actuator axes are placed horizontally on the base plate. ${ }^{(3)}$ Zhao et al. ${ }^{(4)}$ used the first type of three-axis platform combined with the $X-Y$ platform for the physical stage and a prototype micromachine tool. He et al. ${ }^{(5)}$ presented a series-parallel hybrid platform that employed an abrasive belt as the tool and integrated the measuring equipment to achieve in situ measurement for blade polishing. They studied control strategies to solve processing parameters aiming at the characteristics of the alternate measurement and processing in polishing. According to singularity analysis of the parallel mechanism, the platform structure parameters were appropriately optimized to avoid the singular position. Fan et al. ${ }^{(6)}$ used the second type of three-axis platform to conduct inverse kinematics derivation and then analyzed a sensitivity model for a spindle platform that was affected by structural parameters. A three-dimensional (3D) laser ball bar was also employed to construct a model for identifying mechanism errors. ${ }^{(7)}$ Cheng et al. ${ }^{(8)}$ used the third type of three-axis platform combined with an $X-Y$ platform to investigate the calibration of the three-axis platform and apply it to concentric drilling with multiple surfaces and angles. Kanaan et al. ${ }^{(9)}$ performed kinematic analysis of a serial-parallel machine tool: the VERNE machine. This machine is a five-axis machine tool designed by Terrier et al., ${ }^{(10)}$ it combines a 3DOF parallel module and a two-degree-offreedom serial tilting table. Guo et al. ${ }^{(11)}$ proposed the mechanical structure, kinematics, dynamics, and control system of a new five-degree-of-freedom hybrid robot manipulator. Lue et al. ${ }^{(12)}$ proposed the system structure and contour tracking for a hybrid motion platform. The reconfigurable parallel five-axis machine center can be applied to machining for various purposes. $^{(4-13)}$ 
The realization of excellent machining quality requires not only high-quality machines but also computer-aided design (CAD) or computer-aided manufacturing (CAM) software with powerful functions to assist in the calculation of the cutting trajectories in the machining area. Developing comprehensive CAM software for machining is difficult and requires repeated testing and verification. Typically, a postprocessor is used to convert cutting tool path files (produced from a CAD/CAM system) into program codes that can be accepted by machining centers. $^{(14,15)}$ This is more difficult for five-axis machining software-because different machining centers feature different kinetics - and particularly difficult for hybrid machining centers. $^{(16-18)}$ The key technology of five-axis machining is the effect of the rotational tool center point. $^{(19,20)}$ Control of the tool center point ensures that the contact point of the tool center, cutting tool, and workpiece surface remains unchanged. The tool center point is located on the normal line of the actual contact point between the cutting tool and the workpiece surface, and the shank rotates around the tool center point. Because the numerical control (NC) of a five-axis machining center does not have a real-time trajectory system, CAM programming and postprocessing are required to plan the trajectory of the cutting tool in advance. Similarly, if the machining center or the cutting tool changes, CAM programming and postprocessing must be conducted again. Cheng and Lin used two PCI-8134 four-axis servo motor control cards to control a reconfigurable five-axis machine tool (RFMT). ${ }^{(21)}$ One card controlled a 3-PRS parallel three-axis motion platform, and the other controlled the $X-Y$ motion table. The linkage mechanism was driven to perform machining. The servo motor encoder transmitted a pulse back to the PCI-8134 four-axis servo motor control cards, and LabVIEW was used for monitoring. Although the RFMT could be controlled, there was a delay. Therefore, in this study, the delay will be reduced by using an OPEN computer numerical control (CNC) system to control an RFMT.

The main architecture of the OPEN CNC system includes a motion controller, a humanmachine interface (HMI), NC machining codes ( $G, M, S$, and $T$ ), and motion login controller (MLC). Figure 2 shows the main architecture of OPEN CNC. In this research, a 10B milling machine controller from SYNTEC was used as the development controller of the RFMT.

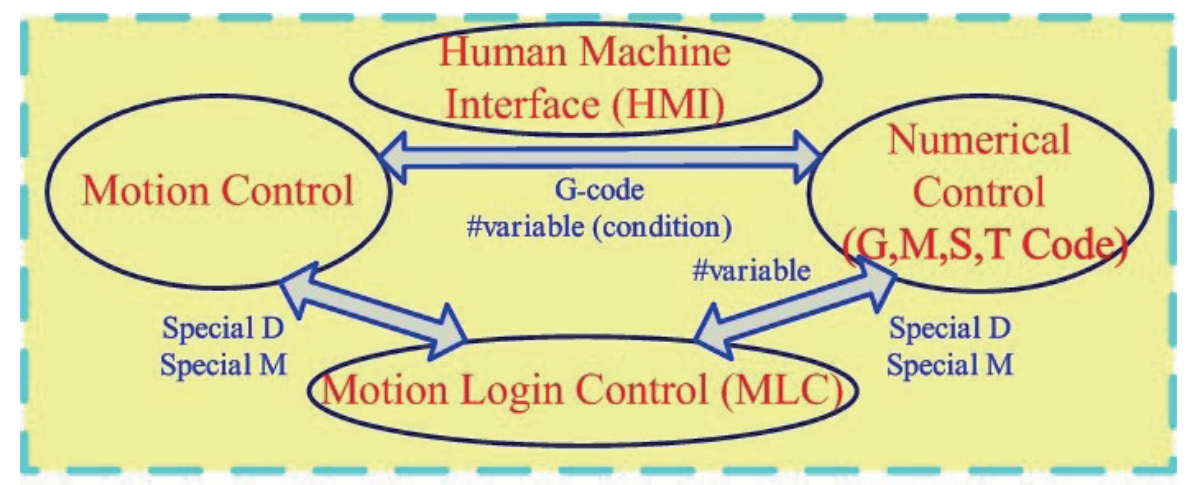

\section{OPEN CNC Architecture}

Fig. 2. (Color online) OPEN CNC architecture. 
To enable the application of the parallel five-axis machining center to more machining situations, this study was conducted using the commercial CAM software Siemens NX. Specifically, the illustration software Autodesk Inventor was employed to illustrate 3D machining workpieces, which were then input into Siemens NX. A suitable machining cutting tool was selected, and the five-axis $\mathrm{NC}$ machining codes for the complex surfaces were calculated. The derived five-axis NC machining codes were then input into the programmed graphical monitoring software LabVIEW to convert the five-axis postprocessor data. Subsequently, the $X, Y$, and $Z$ values of the output NC machining codes of the parallel fiveaxis machining center were extracted and input into Autodesk Inventor for verification. After the values were verified, the machining point was converted into the drive value for each axis of the reconfigurable parallel five-axis machining center and then input into the five-axis simultaneous machining center to finish the machining. Finally, rough and finishing methods were used to cut a concave circle to verify the accuracy.

\section{Mechanism Analysis}

Figure 1 shows the reconfigurable parallel five-axis machining center used in this study, which was composed of a 3DOF parallel platform and an $X-Y$ platform. The inverse kinematics for the parallel three-axis motion platform are derived in the following sections. Please refer to Table 1 for all mathematical symbols.

Table 1

Mathematical symbols.

\begin{tabular}{|c|c|c|}
\hline Symbol & Unit & Description \\
\hline$\alpha$ & deg & Angle of rotation along the $X$-axis \\
\hline$\beta$ & deg & Angle of rotation along the $Y$-axis \\
\hline$Z$ & $\mathrm{~mm}$ & Height \\
\hline$U$ & & Movable platform center point \\
\hline$B$ & & Base center point \\
\hline$U U i$ & & $\begin{array}{l}\text { Coordinate from the movable platform center point to the center of the universal joint } \\
{[X U Y U Z U]}\end{array}$ \\
\hline$B B i$ & & Coordinate from the base center point to the center of the drive shaft $[X B Y B Z B]$ \\
\hline$\theta i$ & deg & Angle between two axes \\
\hline$B U i$ & & UUi coordinate system converted into the coordinate system of the base. \\
\hline$B T U$ & & Movable platform converted into the coordinate matrix of the base \\
\hline$B R U$ & & Conversion equation for roll, pitch, and yaw angles \\
\hline BUix & & $\begin{array}{l}X \text {-axis value in the coordinate system of the base converted from the coordinate system } \\
\text { of the movable platform }\end{array}$ \\
\hline BUiy & & $\begin{array}{l}Y \text {-axis value in the coordinate system of the base converted from the coordinate system } \\
\text { of the movable platform }\end{array}$ \\
\hline BUiz & & $\begin{array}{l}Z \text {-axis value in the coordinate system of the base converted from the coordinate system } \\
\text { of the movable platform }\end{array}$ \\
\hline$D i$ & $\mathrm{~mm}$ & Length of the connecting rod \\
\hline $\mathrm{Li}$ & $\mathrm{mm}$ & Distance between the center point of the base and the drive shaft \\
\hline$T 1$ & & Conversion equation for roll, pitch, and yaw angles \\
\hline$T 2$ & & Movable platform converted into the coordinate matrix of the base $(Z)$ \\
\hline$T 3$ & & Movable platform converted into the coordinate matrix of the base $(T L)$ \\
\hline$T$ & & $\begin{array}{l}Z * T L \text { in the coordinate system of the base converted from the movable platform } \\
\text { coordinate system }\end{array}$ \\
\hline
\end{tabular}




\subsection{Inverse kinematics of mechanism}

First, the inverse kinematics of the three-axis mechanism were derived to control the trajectory, namely, to control the extension from the center point of the movable platform $(U)$ to the three-axis drive shaft. The inverse kinematics can be used to convert the attitude of the movable platform into the amount of extension of the three actuator axes. The locations of the various connection points on the base and the movable platform are presented in Fig. 3.

$$
\begin{gathered}
{ }^{U} U_{i}=r \cdot\left[\begin{array}{llll}
\cos \theta_{i} & \sin \theta_{i} & 0 & 1
\end{array}\right]^{T} ; i=1-3 \\
{ }^{B} B_{i}=R \cdot\left[\begin{array}{llll}
\cos \theta_{i} & \sin \theta_{i} & 0 & 1
\end{array}\right]^{T}
\end{gathered}
$$

Here, ${ }^{U} U_{i}$ and ${ }^{B} B_{i}$ should be separately defined. Superscripts $U$ and $B$ denote the coordinate system of the movable platform and the base platform; $U$ and $B$ denote the movable platform and the base platform, respectively, and the subscript $i$ is the number of each drive shaft. Here, $\gamma$ is the radius of the circumcircle of the movable platform and $R$ is the radius of the circumcircle of the base platform.
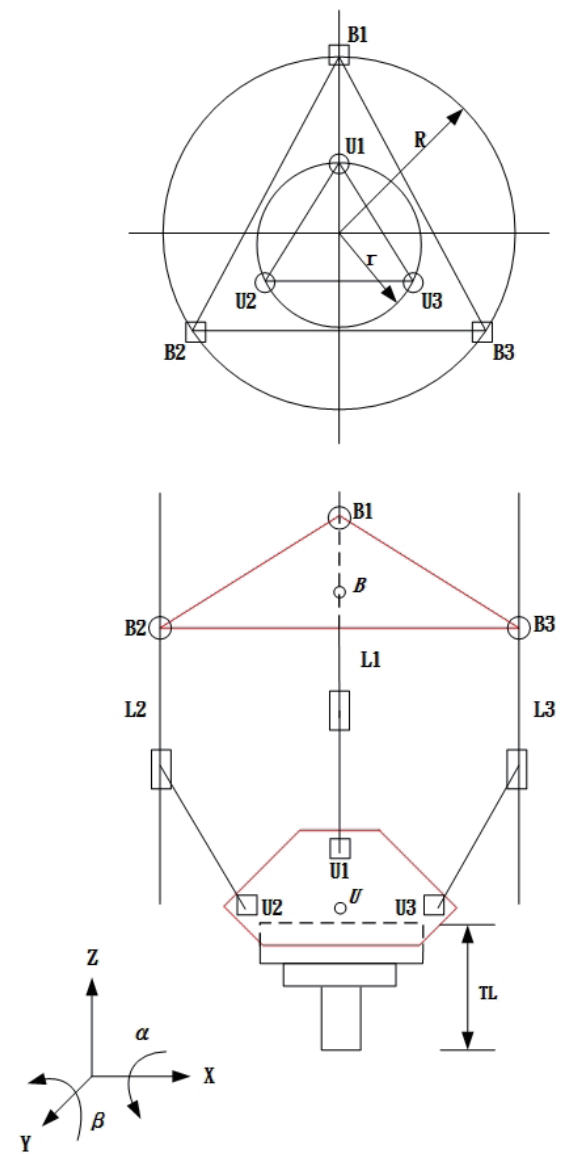

Fig. 3. (Color online) Parallel three-axis mechanism. 


$$
\theta=\frac{2 \pi}{3}(i-1) ; i=1-3
$$

The coordinate values of all points on the movable platform and the base $\left(X_{B}, Y_{B}, Z_{B}\right)$ are expressed as

$$
{ }^{B} U_{i}=\left[{ }^{B} T_{U}\right]\left[{ }^{U} U_{i}\right] ; i=1-3,
$$

where

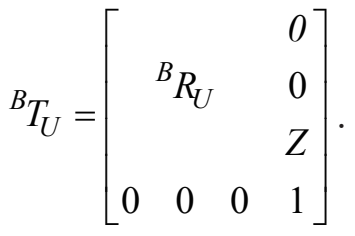

The coordinate points on the movable platform were converted into the coordinate system of the base $(B)$ using the roll, pitch, and yaw angles and $\gamma=0$. Therefore, the rotation coordinate is expressed as

$$
{ }^{B} R_{U}=R_{y, \beta} \cdot R_{x, \alpha}=\left[\begin{array}{ccc}
\cos \beta & \sin \beta \cdot \sin \alpha & \sin \beta \cdot \cos \alpha \\
0 & \cos \alpha & -\sin \alpha \\
-\sin \beta & \cos \beta \cdot \sin \alpha & \cos \beta \cdot \cos \alpha
\end{array}\right] .
$$

According to Eq. (4), the coordinate values of the connection points on the movable platform that has been converted into the coordinate system of the base are

$$
{ }^{B} U_{i}={ }^{B} T_{U} \cdot{ }^{U} U_{i}=\left[\begin{array}{c}
{ }^{B} U_{i x} \\
{ }^{B} U_{i y} \\
{ }^{B} U_{i z}
\end{array}\right]=\left[\begin{array}{c}
r \cdot \cos \theta_{i} \cdot \cos \beta+r \cdot \sin \theta_{i} \cdot \sin \beta \cdot \sin \alpha \\
r \cdot \sin \theta_{i} \cdot \cos \alpha \\
Z-r \cdot \cos \theta_{i} \cdot \sin \beta+r \cdot \sin \theta_{i} \cdot \sin \alpha
\end{array}\right] .
$$

The connecting rods were expressed in the form of vectors:

$$
\vec{D}_{i}={\overrightarrow{U_{i} B_{i}}}
$$

The length of each connecting rod is

$$
D_{i}={ }^{B} U_{i}-{ }^{B} B_{i},
$$

where $D_{i}$ signifies the length of the connecting rod (fixed value) and ${ }^{B} B_{i Z}=0$; 


$$
D_{i}^{2}=\left({ }^{B} U_{i x}-{ }^{B} B_{i x}\right)^{2}+\left({ }^{B} U_{i y}-{ }^{B} B_{i y}\right)^{2}+\left({ }^{B} U_{i z}-{ }^{B} B_{i z}\right)^{2},
$$

where

$$
D_{i}^{2}=\left({ }^{B} U_{i x}-{ }^{B} B_{i x}\right)^{2}+\left({ }^{B} U_{i y}-{ }^{B} B_{i y}\right)^{2}+{ }^{B} U_{i z}{ }^{2}
$$

Expanding the brackets in Eq. (11) gives

$$
{ }^{B} B_{i z}={ }^{B} U_{i z}-\sqrt{D_{i}^{2}-\left({ }^{B} U_{i x}-{ }^{B} B_{i x}\right)^{2}+\left({ }^{B} U_{i y}-{ }^{B} B_{i y}\right)^{2}} .
$$

\subsection{Parallel five-axis postprocessing equation}

The five-axis postprocessing equation was derived from Fig. 4 and is expressed as follows.

$$
T_{1}=\left[\begin{array}{ccc}
\cos \beta & \sin \beta \cdot \sin \alpha & \sin \beta \cdot \cos \alpha \\
0 & \cos \alpha & -\sin \alpha \\
-\sin \beta & \cos \beta \cdot \sin \alpha & \cos \beta \cdot \cos \alpha
\end{array}\right]
$$

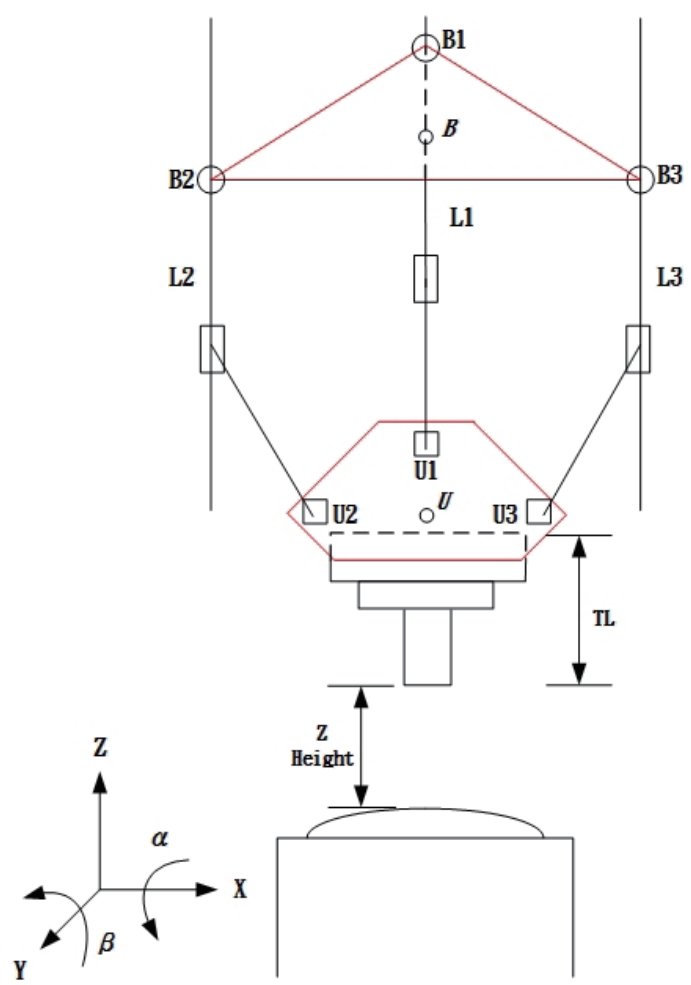

Fig. 4. (Color online) Compensation for cutting tool length and height of $Z$-axis on parallel three-axis platform. 
Substitute Eq. (13) into Eq. (14):

$$
T_{2}=\left[\begin{array}{llll} 
& & & 0 \\
& T_{1} & & 0 \\
& & & Z \\
0 & 0 & 0 & 1
\end{array}\right]
$$

where $T L$ indicates the total length of the cutting tool. The $T_{3}$ matrix is expressed as

$$
T_{3}=\left[\begin{array}{cccc}
1 & 0 & 0 & 0 \\
0 & 1 & 0 & 0 \\
0 & 0 & 1 & T L \\
0 & 0 & 0 & 1
\end{array}\right]
$$

The current turning platform was added onto the surface of the cutting tool, and $T_{2}$ was multiplied by $T_{3}$ to obtain

$$
T=T_{2} T_{3}=\left[\begin{array}{cccc} 
& & & 0 \\
& T_{1} & & 0 \\
& & & Z \\
0 & 0 & 0 & 1
\end{array}\right] \cdot\left[\begin{array}{cccc}
1 & 0 & 0 & 0 \\
0 & 1 & 0 & 0 \\
0 & 0 & 1 & T L \\
0 & 0 & 0 & 1
\end{array}\right] .
$$

Expanding Eq. (16) gives

$$
T=\left[\begin{array}{cccc}
\cos \beta & \sin s \alpha \cdot \sin \beta & \cos \alpha \cdot \sin \beta & T L \cdot \cos \alpha \cdot \sin \beta \\
0 & \cos \alpha & -\sin \alpha & T L \cdot(-\sin \alpha) \\
-\sin \beta & \sin \alpha \cdot \cos \beta & \cos \alpha \cdot \cos \beta & T L \cdot \cos \alpha \cdot \cos \beta+Z \\
0 & 0 & 0 & 1
\end{array}\right]
$$

The derived equations were integrated to obtain the following parallel five-axis postprocessing equations.

$$
\begin{gathered}
X \rightarrow T L \cdot c \alpha \cdot s \beta \\
Y \rightarrow T L \cdot(-s \alpha) \\
Z \rightarrow T L \cdot c \alpha \cdot c \beta+Z
\end{gathered}
$$




\section{Experimental Setting}

\subsection{Calibration of parallel five-axis machining center}

Because the installation relationship of the parallel spindle and the length of the installation component may be different, the height of the axes may also differ. Therefore, the machining center must be calibrated at the beginning of the experiment. The steps for calibrating the spindle of the parallel five-axis machining center are as follows:

Step 1. Connect a gyroscope to the computer and place it on a horizontal platform; set the horizontal value to 0 .

Step 2. Install the gyroscope on the parallel movable platform, as shown in Fig. 5.

After reading the figures of the gyroscope, the heights of the three axes are manually calibrated and the movable platform is adjusted to be horizontal.

\subsection{Experimental framework}

Figure 6 shows a flowchart of the experiment. Autodesk Inventor was used to illustrate a 3D machining workpiece, which was then input into Siemens NX CAM software to obtain a fiveaxis NC code. The code was subsequently incorporated into the postprocessing equation, which was generated using LabVIEW, to determine the NC code for the parallel five-axis machining center. After conversion, the values of each axis were stored in the OPEN CNC system, and then controlled using $\mathrm{NC}$ machining codes $(G, M, S$, and $T)$ one by one. In this research, a 10B milling machine controller from SYNTEC was used as the development controller of the RFMT. Figure 7 shows the experimental system architecture.

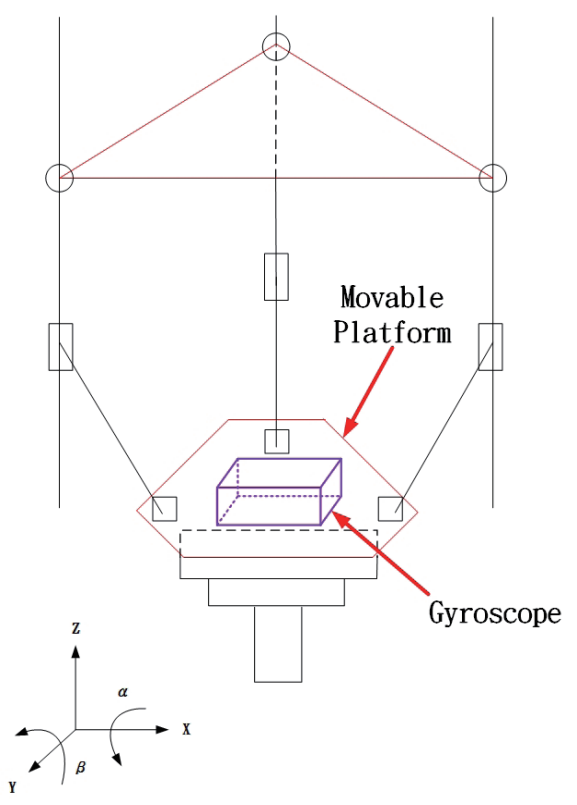

Fig. 5. (Color online) Gyroscope position. 


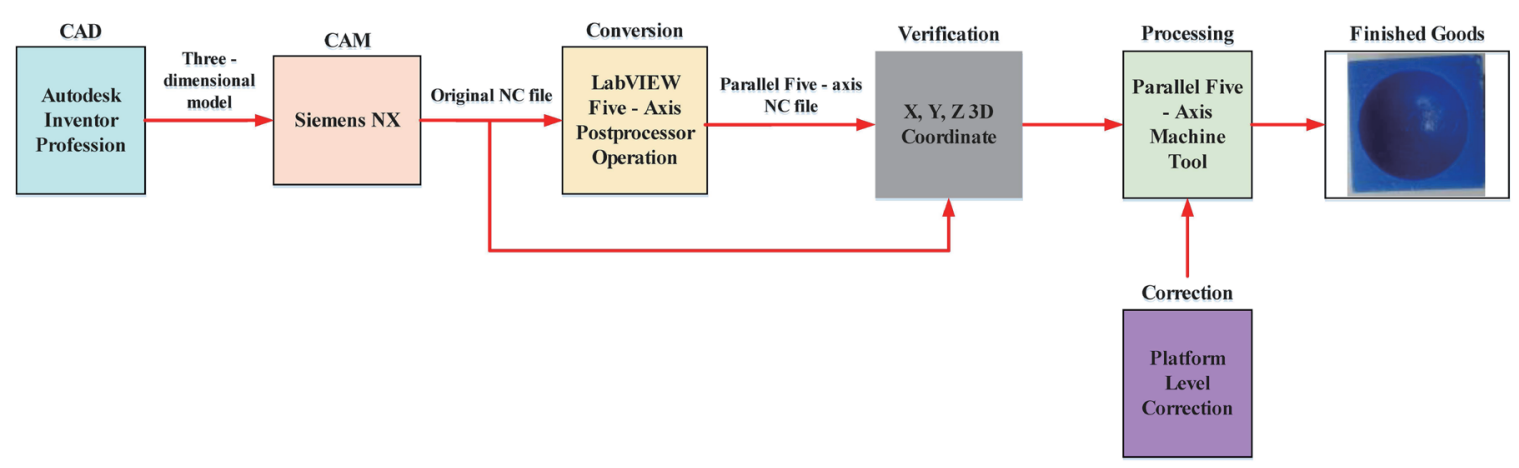

Fig. 6. (Color online) Experiment flowchart.

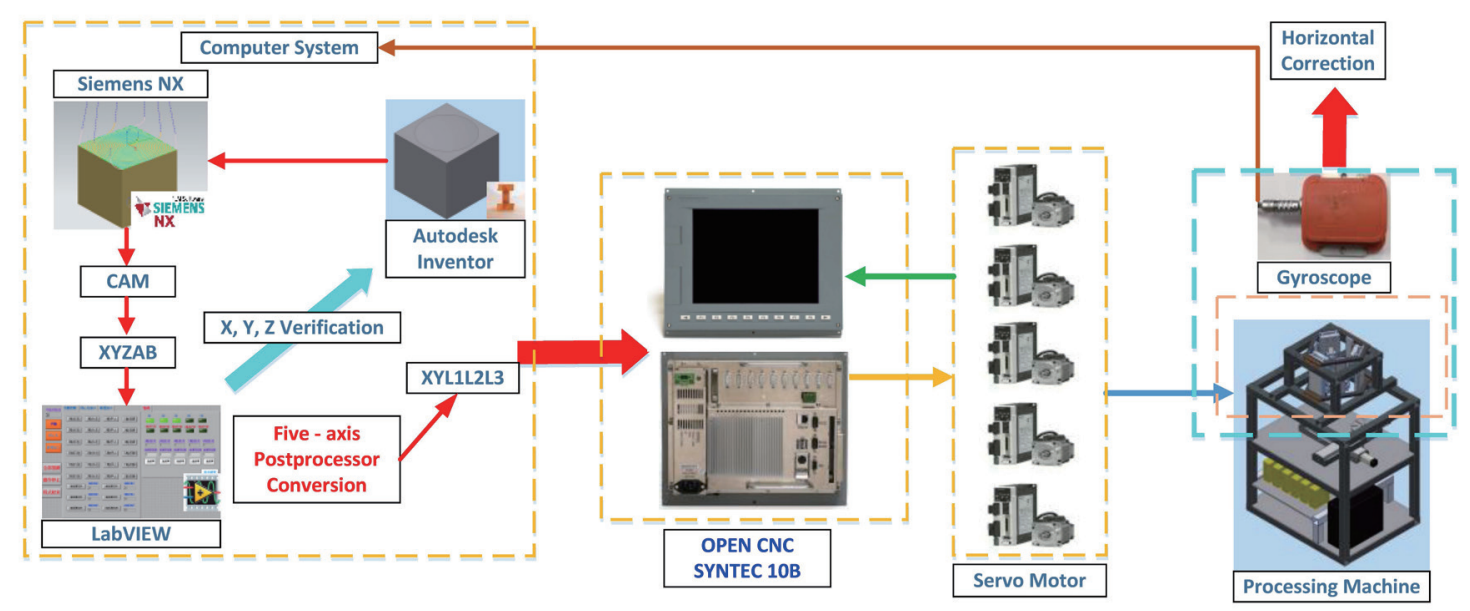

Fig. 7. (Color online) Experimental system architecture.

\subsection{Five-axis postprocessor conversion}

Five-axis NC machining codes $(X, Y, Z, A$, and $B)$ were obtained from NX CAM software, and a program was written in LabVIEW to read the source NC machining code. We use the serial number $\mathrm{N}$ in the $\mathrm{NC}$ machining code to read the $X, Y, Z, A$, and $B$ of each line, and enter the total length of the cutting tool and the height of the $Z$-axis for postprocessing. In the fiveaxis postprocessor conversion program, the values of $Z, A$, and $B$ were calculated using inverse kinematics to find the three axis lengths L1, L2, and L3. The $X$ and $Y$ values control the $X-Y$ platform. In addition, in the study, M12 was also set to three axes (L1, L2, and L3) in OPEN CNC's MLC. Therefore, the HI could control simultaneous or independent movement. Figure 8 shows the five-axis postprocessor conversion flowchart.

\subsection{Steps of parallel five-axis machining center experiment}

The steps for the application of five-axis postprocessor conversion to the parallel five-axis machining center are as follows: 
Step 1 Use LabVIEW to write a parallel three-axis motion platform equation.

Step 2 Use LabVIEW to write a five-axis postprocessor conversion program.

Step 3 Plan the HMI and monitoring screens for LabVIEW.

Step 4 Use Autodesk Inventor to draw a 3D machining workpiece.

Step 5 Input the produced 3D workpiece into the Siemens NX 10 CAM software to generate five-axis $\mathrm{NC}$ machining codes.

Step 6 Input the obtained NC machining codes into the LabVIEW five-axis postprocessor.

Step 7 Output the NC machining codes of the parallel five-axis machining center; extract the position values of $X, Y$, and $Z$; and input the values into Autodesk Inventor for verification.

Step 8 Use the OPEN CNC system to control the parallel five-axis machining center for the cutting experiment to verify the feasibility and accuracy of the machining center.

\section{Results and Discussion}

\subsection{Results of calculation of original concave circle machining}

To verify the machining precision and usability of this machine, we used the postprocessing program to transfer the $\mathrm{NC}$ machining code using five-axis machining software to determine the processing path; the $Z, \alpha$, and $\beta$ values of the NC program are used to convert each axis value of a three-axis motion platform through inverse kinematics as shown in Figs. 6-8. Finally, two different tools were used to cut a concave circle in a wax block by a roughing method and finishing method to verify the accuracy.

The five-axis machining center comprised three translational axes and two rotational axes. In the Siemens NX $10 \mathrm{CAD} / \mathrm{CAM}$ software, the reconfigurable five-axis machining center proposed in this study was set to be an orthogonal, spindle SBSA-type five-axis machining center. An R5 ball-nose cutter was selected as the cutting tool, and the toward point was

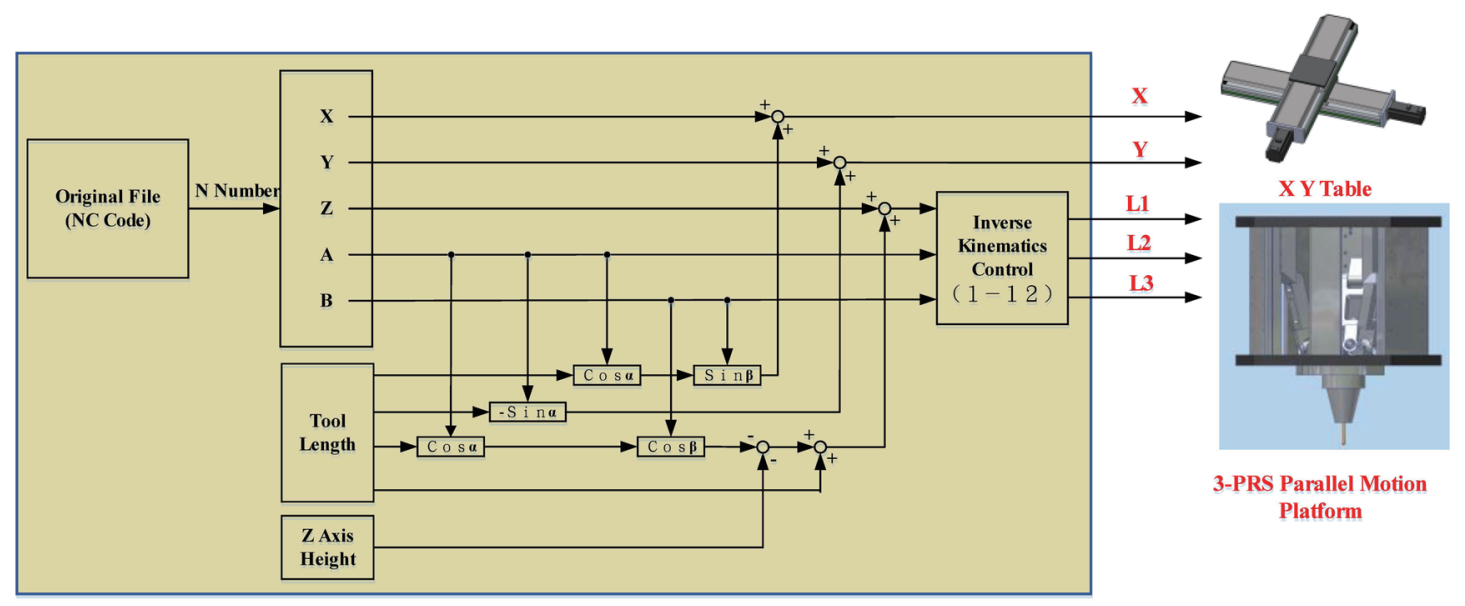

Fig. 8. (Color online) Five-axis postprocessor conversion flowchart. 
chosen. The shank swung on a point $150 \mathrm{~mm}$ above the $Z$-axis for reciprocating machining to meet the requirement for the machining center workspace based on the rotation angle. Figure 9 is a dimension drawing of concave circle processing. A side-cutting edge was used for machining. This is mainly because the center point of the ball-nose cutter is only a quiescent point without a cutting edge and is therefore unsuitable for the machining point.

Figure 10 shows the $X Y Z$ values of 352 five-axis machining points calculated by NX 10 CAM. The NX 10 software was used to draw a spatial diagram of all machining points to try to extract the maximum boundary value on both sides and calculate the maximum diameter. The maximum diameter is $15.53 \mathrm{~mm}$. The cutting depth is $12.40 \mathrm{~mm}$, and it can be compared with the processing diagram shown in Fig. 9. Figure 11 shows the $X, Y$, and $Z$ values of all five-axis machining points drawn using MATLAB software. Figure 12 shows the path change values of the $A B$ axis of the five-axis machining point. Figure 13 shows the $X Y$ path of the original machining program. Figure 14 is a 3D image of the $X Y Z$ path for the original machining program. Figure 15 is a 3D image of the angle path for the original machining program. Figures $10-15$ show the pictures of the original concave circle machining.

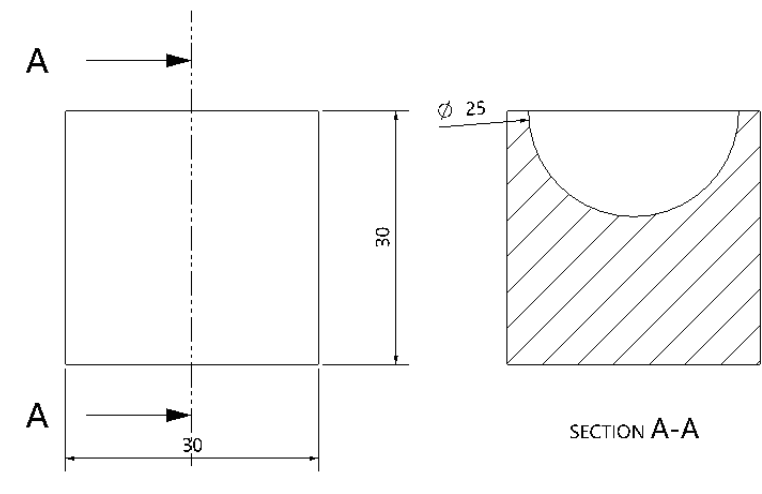

Fig. 9. Dimensions for concave circle processing.
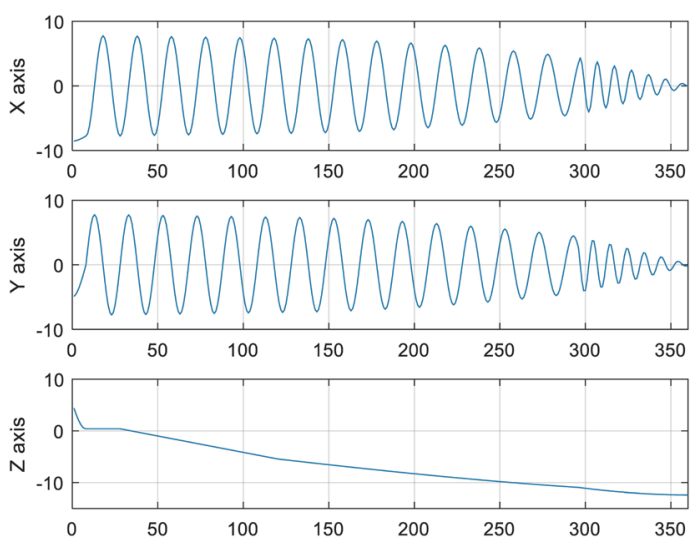

Fig. 11. (Color online) $X Y Z$ path of original machining program.

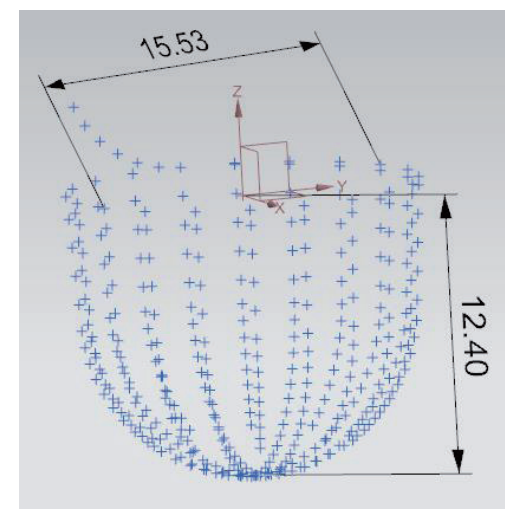

Fig. 10. (Color online) Spatial diagram of original concave circle after rough machining.
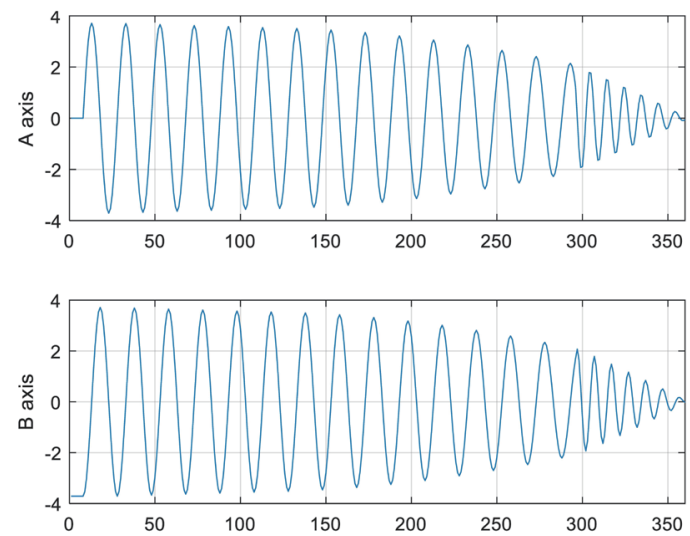

Fig. 12. (Color online) Angle path of original machining program. 


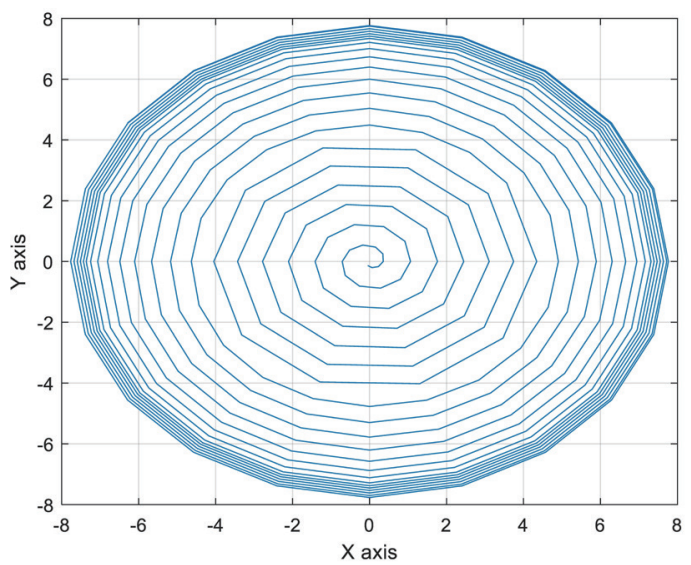

Fig. 13. (Color online) $X Y$ path of original machining program.

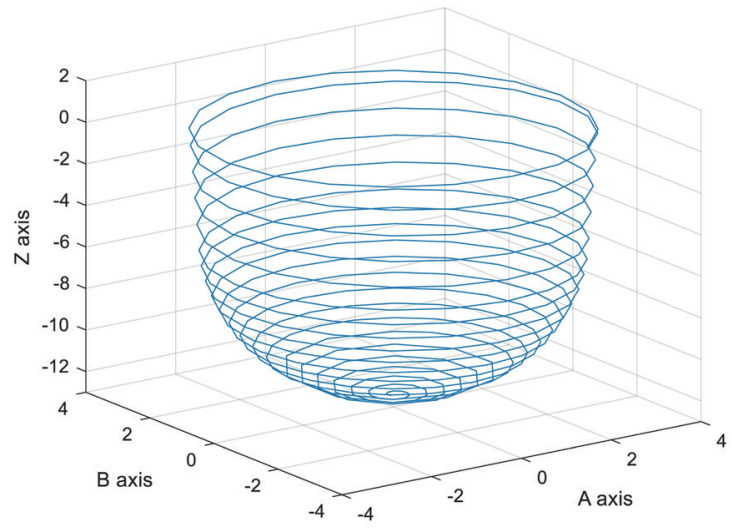

Fig. 15. (Color online) 3D image of angle path of original machining program.

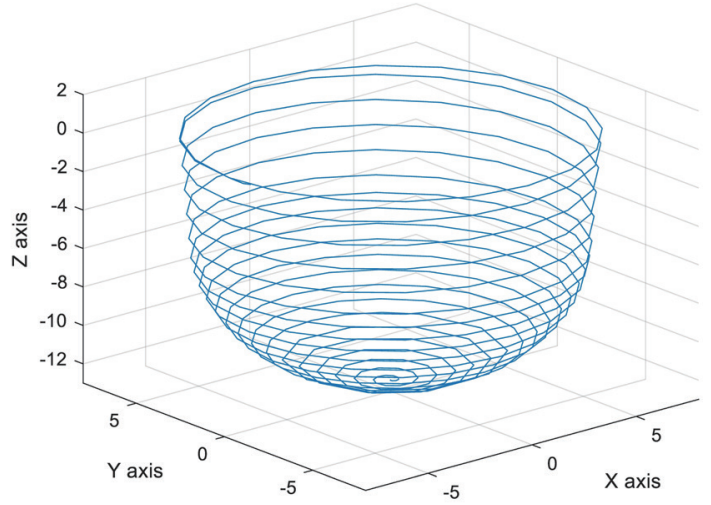

Fig. 14. (Color online) 3D image of $X Y Z$ path of original machining program.

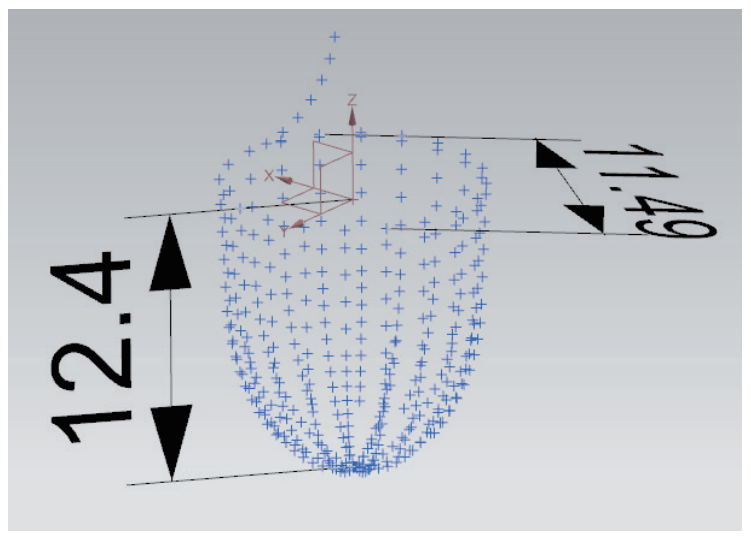

Fig. 16. (Color online) Spatial diagram of converted concave circle after rough machining.

\subsection{Results of calculation of converted concave circle machining}

Figure 16 is a spatial diagram of the $X Y Z$ values of the five-axis machining points converted using the flowchart in Fig. 8, with a maximum distance of $11.49 \mathrm{~mm}$ between the two points and a maximum cutting depth of $12.40 \mathrm{~mm}$. In Figs. 10 and 16, the maximum distance between the top and bottom points was measured and the tool must cut the same depth. The maximum diameter was reduced from 15.53 to $11.49 \mathrm{~mm}$ because the three-axis table rotates the cutting tool, so the $X Y$ value is reduced.

Figure 17 shows the lengths of the three parallel axes after converting the machining program. Figure 18 shows the path of the $X Y$ axis after converting the machining program on each axis. Figure 19 shows the $X Y$ coordinate diagram after converting the processing program. Figure 20 is a $3 \mathrm{D}$ image of the ABL1 path after converting the machining program. Figure 21 is a spatial diagram of 700 cutting points for the original concave circle finishing, where the maximum diameter of the circle was $15.47 \mathrm{~mm}$ and the maximum cutting depth was $12.50 \mathrm{~mm}$. Figure 22 is a spatial diagram of the converted concave circle after finishing machining. 

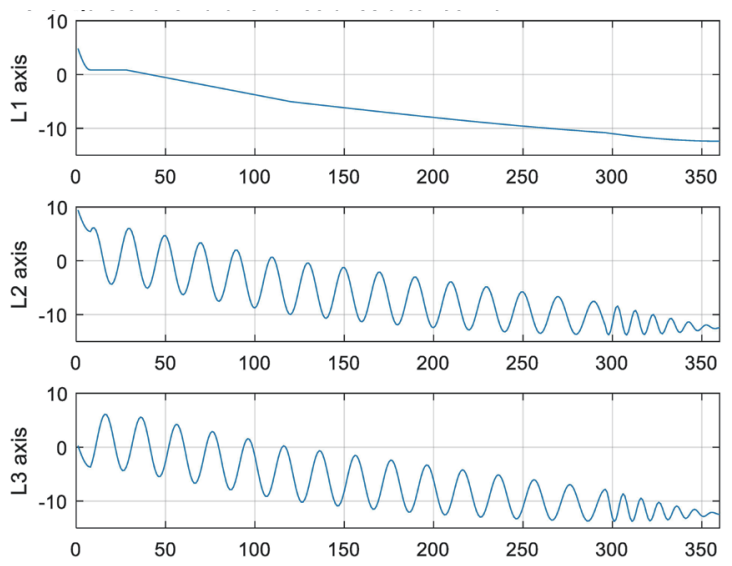

Fig. 17. (Color online) Lengths of three parallel axes after converting machining program.

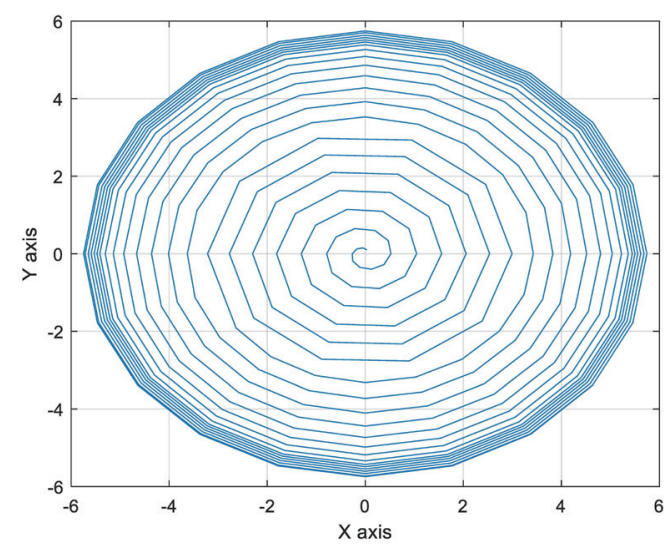

Fig. 19. (Color online) $X Y$ path after converting machining program.

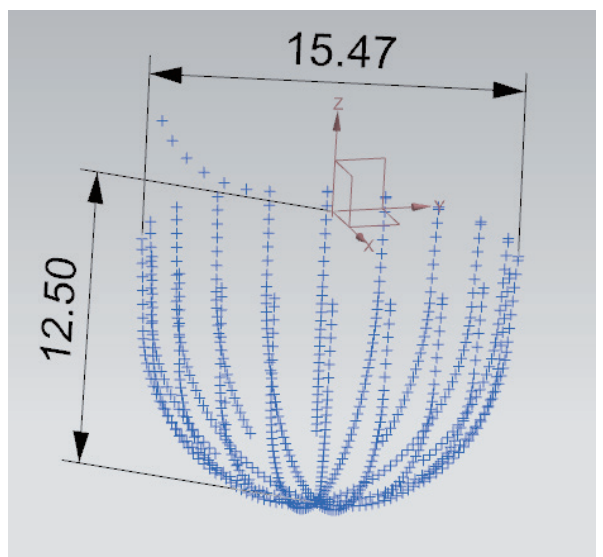

Fig. 21. (Color online) Spatial diagram of original concave circle after finishing machining.
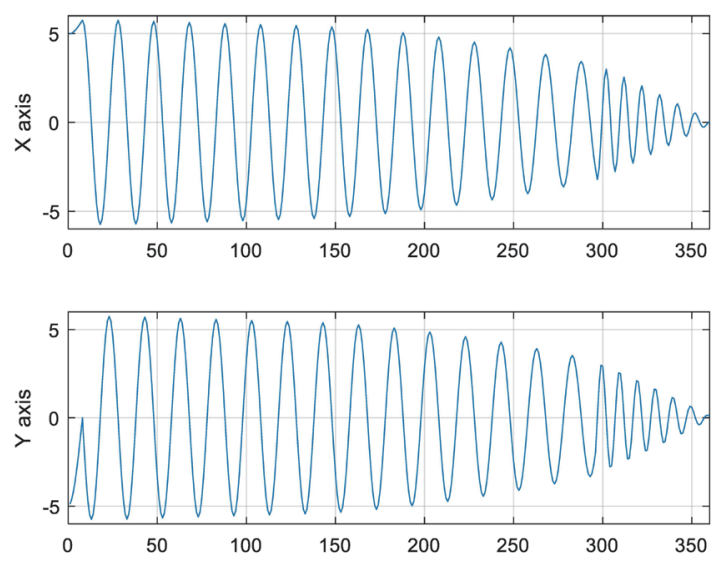

Fig. 18. (Color online) Path of $X Y$ axis after converting machining program.

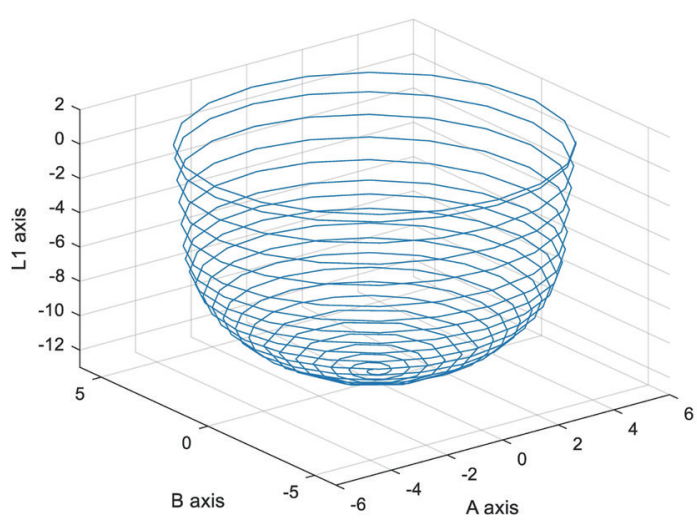

Fig. 20. (Color online) 3D image of ABL1 path after converting machining program.

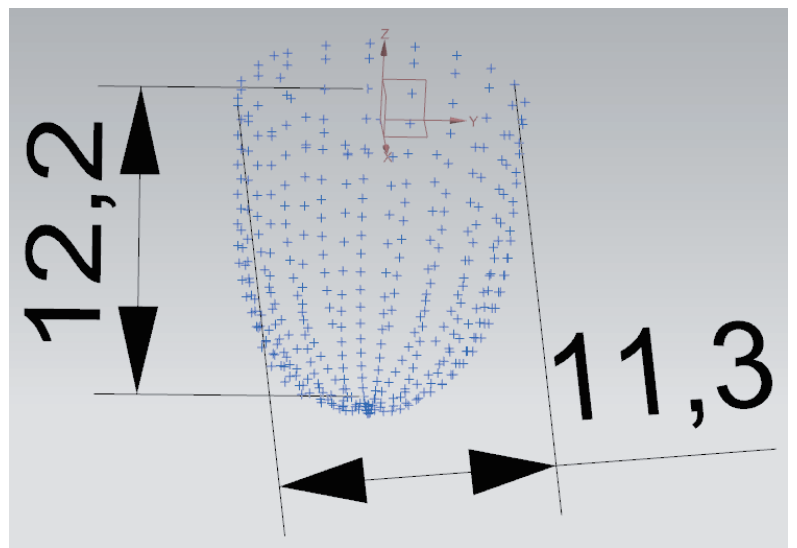

Fig. 22. (Color online) Spatial diagram of converted concave circle after finishing machining. 

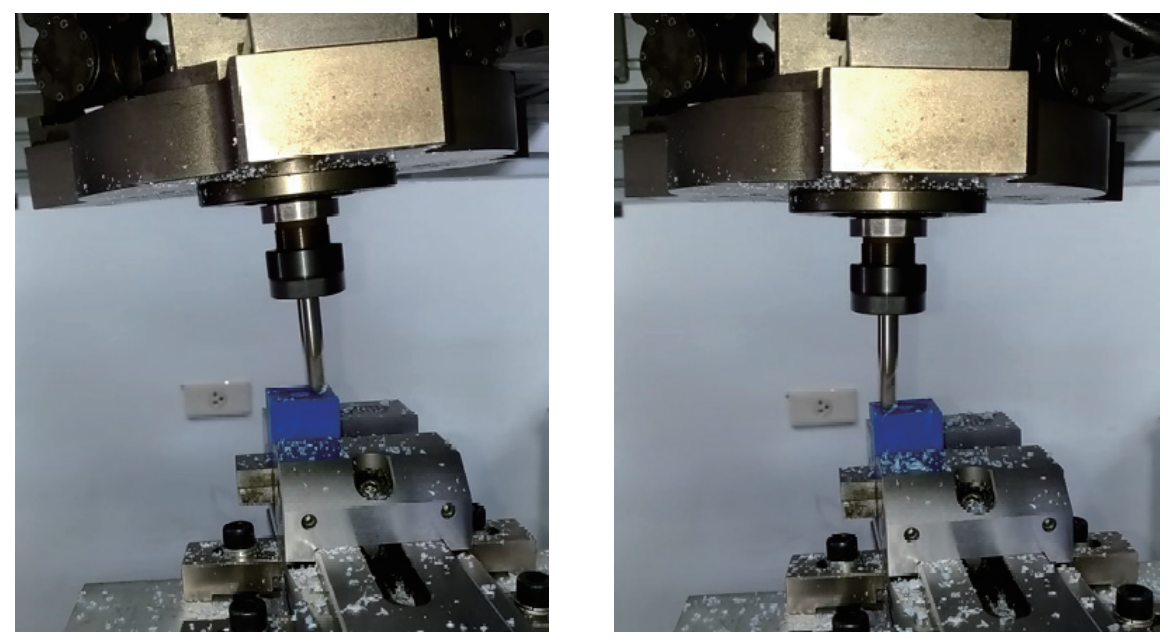

Fig. 23. (Color online) Processing diagram of actual cutting concave circle.

Figures 16-22 show the images of converted concave circle machining points. Videos were taken during the experiment, but only screenshots are shown to illustrate the phenomena in the experimental processing. Figure 23 shows the actual cutting concave circle processing status. These videos are available to readers by email.

\section{Conclusions}

We used an OPEN CNC system to develop a reconfigurable five-axis machine tool for system integration research. In this study, the HMI of OPEN CNC was written, and the programmable logic controller (PLC) architecture of OPEN CNC was used to control three-axis simultaneous movement or individual drives. The main contributions of this research are as follows:

1. We derive inverse kinematics and calculate the five-axis postprocessing equation for the parallel three-axis platform of the parallel five-axis machining center.

2. The experimental results (Figs. 16 and 21) show that the derived equation and calculation are correct. After verifying the value of machining points, the processing point is converted to the drive value of each axis of the reconfigurable parallel five-axis machining center and then input to the five-axis simultaneous machining center to complete the processing.

3. In this study, the rough and finishing methods are used to cut a concave circle to verify the accuracy.

4. We demonstrate that the parallel five-axis postprocessor developed using LabVIEW can convert the NC machining codes generated by Siemens NX 10 CAM software into codes suitable for the parallel five-axis machining center used in this study to conduct actual machining.

5. The experimental results of the actual processed product are almost the same as the 3D model (Fig. 9), which verified that the proposed method possesses high accuracy. 


\section{References}

1 Y. M. Cheng and Y. S. Chen: J. Mech. Eng. Autom. 3 (2013) 14. 10.17265/2159-5275/2013.01.003 https://doi/ org/10.17265/2159-5275/2013.01.003

2 Y. M. Cheng, P. Y. Chen, W. L. Huang, and H. H. Wu: J. Prof. Mech. Eng. 6 (2013) 53. https://doi/org/10.6208/ JPME-2013-12-08

3 Y. M. Cheng: Appl. Mech. Mater. 52-54 (2011) 517. https://oi/org/10.4028/www.scientific.net/AMM.5254.517

4 G. Zhao, Y. Deng, W. L. Xiao, and Y. Z. Liu: J. Adv. Mech. Des. Syst. 11 (2017) 1. https://doi/org/10.1299/ jamdsm.2017jamdsm0035

5 Q. W. He, J. Zhao, M. Feng, C. P. Zhang, and H. Chen: Int. J. Adv. Manuf. Technol. 102 (2019) 265. https://doi. org/10.1007/s00170-018-3064-9

6 K. C. Fan, H. Wang, J. W. Zhao, and T. H. Chang: Int. J. Mach. Tools Manuf. 43 (2003) 1561. https://doi. org/10.1016/S0890-6955(03)00202-5

7 H. Wanga and K. C. Fan: Int. J. Mach. Tools Manuf. 44 (2004) 1171. https://doi.org/10.1016/ j.ijmachtools.2004.04.005

8 Y. M. Cheng, W. X. Peng, and A. C. Hsu: Int. J. Adv. Manuf. Technol. 76 (2015) 1253. https://doi/org/10.1007/ s00170-014-6337-y

9 D. Kanaan, P. Wenger, and D. Chablat: Mech. Mach. Theory 44 (2009) 487. https://doi.org/10.1016/ j.mechmachtheory.2008.03.002

10 M. Terrier, M. Giménez, and J. Y. Hascoët: Proc. Inst. Mech. Eng. B J. Eng. Manuf. 219 (2005) 327. https://doi/ org/10.1243/095440505X30177

11 W. J. Guo, R. F. Li, C. Q. Cao, and Y. F. Gao: Adv. Mech. Eng. 8 (2016) 1. https://doi. org/10.1177/1687814016680309

12 C. W. Lue, Y. M. Cheng, and J. H. Chin: Int. J. Adv. Manuf. Technol. 26 (2005) 1388. https://doi/org/10.1007/ s00170-004-2119-2

13 L. Z. Fan, A. Y. Elatta, and X. P. Li: Int. J. Adv. Manuf. Technol. 25 (2005) 730. https://doi.org/10.1007/s00170003-1987-1

14 Q. Liu and T. Huang: Robot Comput. Integr. Manuf. 56 (2019) 140. https://doi.org/10.1016/j.rcim.2018.06.003

15 Y. L. Lai, C. C. Liao, and Z. G. Chao: Robot Comput. Integr. Manuf. 50 (2018) 63. https://doi.org/10.1016/ j.rcim.2017.09.002

16 Y. Ni, Y. Zhang, K. Sun, H. Wang, and Y. Sun: Proc. Inst. Mech. Eng. B. J. Eng. Manuf. 230 (2016) 661. https://doi/org/10.1177/0959651816645687

17 S.-L. Chen, T.-H. Chang, I. Inasaki, and Y.-C. Liu: Int. J. Adv. Manuf. Technol. 20 (2002) 259. https://doi. org/10.1007/s001700200151

18 S.-L. Chen and Y.-C. Liu: Int. J. Adv. Manuf. Technol. 18 (2001) 254. https://doi.org/10.1007/s001700170066

19 Z. W. Lin, J. Z. Fu, H. G. Shen, and W. F. Gan: Int. J. Adv. Manuf. Technol. 74 (2014) 187. https://doi. org/10.1007/s00170-014-5981-6

20 X. Y. Zhou, X. L. Liu, M. Li, Z. and Wang, and X. Meng: Int. J. Adv. Manuf. Technol. 88 (2017) 1505. https:// doi.org/10.1007/s00170-016-8801-3

21 Y. M. Cheng and M. S. Lin: Int. J. Mech. Eng. Technol. 10 (2019) 179. http://www.iaeme.com/ijmet/issues. asp?JType $=$ IJMET\&VType $=10 \&$ IType $=5$ 